\title{
Influence of a back-flow flap on the dynamic stall flow topology
}

\author{
C. C. Wolf - A. D. Gardner - C. B. \\ Merz - S. Opitz
}

Received: date / Accepted: date

\begin{abstract}
Dynamic stall is a major concern for highly loaded helicopter rotors in fast forward flight. The potential of a back-flow flap for dynamic stall reduction is investigated. The flap assembly is mounted on the suction side of a helicopter main rotor-blade airfoil undergoing deep-stall pitch oscillations. Wind-tunnel experiments using high-speed particle image velocimetry were conducted to identify the flow topology and to investigate the flap's method of operation. A phase-averaged proper orthogonal decomposition (POD) is used to identify relevant flow events and to compare test cases with and without flap. The evolution of the large-scale dynamic stall vortex in the initial phases of flow separation is analyzed in detail. The back-flow flap splits the vortex into two smaller vortices and thereby reduces the pitching moment peak. This effect can be described through the eigenmode coefficients of the POD. The study closes with an analysis of different pitching frequencies, which do not affect the flap's method of operation.
\end{abstract}

A version of this paper was presented at the European Rotorcraft Forum (ERF), Lille, France, September 5-8, 2016

C. C. Wolf

DLR Institute of Aerodynamics and Flow Technology, Bunsenstrasse 10, 37073 Göttingen, Germany.

E-mail: Christian.Wolf@DLR.de

A. D. Gardner

DLR Institute of Aerodynamics and Flow Technology, Bunsenstrasse 10, 37073 Göttingen, Germany.

E-mail: Tony.Gardner@DLR.de

C. B. Merz

DLR Institute of Aerodynamics and Flow Technology, Bunsenstrasse 10, 37073 Göttingen, Germany. Present address: Daimler AG, Mercedesstrasse 132, 70327 Stuttgart, Germany.

S. Opitz

DLR Institute of Composite Structures and Adaptive Systems, Lilienthalplatz 7, 38108 Braunschweig, Germany.

E-mail: Steffen.Opitz@DLR.de 
Keywords Helicopter rotor · Wind tunnel · Flow control · Dynamic stall . Experiment

\section{Nomenclature}

\section{Symbols}

a Temporal POD coefficient

c Airfoil model chord (m)

$C_{d} \quad$ Drag coefficient

$C_{l} \quad$ Lift coefficient

$C_{m} \quad$ Pitching moment coefficient

$C_{p} \quad$ Pressure coefficient

$f \quad$ Frequency of pitching $(\mathrm{Hz})$

$k \quad$ Reduced frequency, $k=\pi f c / V_{\infty}$

$m$ POD mode number

$M \quad$ Mach number

$N \quad$ Number of samples

$N^{*} \quad$ Reduced-order cut-off

Re Reynolds number based on $c$

$t \quad$ Time (s)

$u, w \quad$ Velocity in $x$ direction and $z$ direction $(\mathrm{m} / \mathrm{s})$

$\boldsymbol{u}$ Vector of 2-D velocity components, $\boldsymbol{u}=(u, w)$

$V_{\infty} \quad$ Freestream velocity $(\mathrm{m} / \mathrm{s})$

$V_{p} \quad$ In-plane velocity, $V_{p}=\sqrt{u^{2}+w^{2}}(\mathrm{~m} / \mathrm{s})$

$x, z \quad$ Cartesian coordinates $(\mathrm{m})$

$\boldsymbol{x} \quad$ Vector of 2-D coordinates, $\boldsymbol{x}=(x, z)$

$\alpha \quad$ Angle of attack $\left(^{\circ}\right)$

$\beta \quad$ Opening angle $\left({ }^{\circ}\right)$

$\Delta \quad$ Difference of two values

$\lambda$ eigenvalue

$\boldsymbol{\Phi}$ eigenmode, eigen flow field

Abbreviations

1MG '1-meter' wind tunnel at DLR Göttingen

DLR Deutsches Zentrum für Luft- und Raumfahrt

PIV Particle image velocimetry

POD Proper orthogonal decomposition

\section{Introduction}

The dynamic stall phenomenon exerts a strong influence on the performance of highly loaded helicopter rotors in forward and maneuver flight. It is characterized by the development of a dynamic stall vortex, which results in large 
pitching-moment peaks and consequent pitch link loads. After pioneering fundamental investigations on dynamic stall, for example by McCroskey et al. [1, 2] and Carr et al. [3], numerous measures of flow control have been proposed to counter the negative impact of dynamic stall. Amongst other measures, actively deployable vortex generators in the vicinity of the leading edge can delay leading edge-type stall and promote trailing edge-type stall, see Joubert et al. [4]. A variation of several parameters like vortex generator geometry, actuation phase angle and duty cycle, etc. was conducted by Le Pape et al. [5]. It was shown that a reduction of the pitching moment has to be traded off against a penalty in the maximum lift coefficient. A good compromise is, for example, found for pitching moment reductions in the range of $30 \%$. Heine et al. [6] report experimental results for passive disturbance generators with different geometries, which were attached to an airfoil in the vicinity of the leading edge. The pitching moment coefficient $C_{m}$ during dynamic stall was reduced by up to $42 \%$. Furthermore, the authors emphasize that the disturbance generators also have a positive effect on the lift and drag characteristics during stalled phases of the pitch cycle. On the downside, the non-retractable devices increase the drag during attached flow conditions, affecting the rotor's power requirement in flight conditions without dynamic stall. Other measures of flow control applied to dynamic stall phenomena include air jets with zero net-mass flux $[7,8]$, plasma actuators [9], or morphing leading edge geometries [10]. Gerontakos and Lee [11] applied a trailing edge flap for dynamic stall flow control. It was shown that the pressure distribution on the flap can counteract the pitching moment peak if the flap is deflected upward during dynamic stall, but the formation of the stall vortex itself is not suppressed. Back-flow flaps are inspired by nature, they can be found in the form of pop-up feathers on bird wings which delay stall. A technical application was proposed by Meyer [12]. With a view to a glider aircraft, this type of flap demonstrated an increased maximum lift of about $10 \%$ [13]. The flaps were attached to the upper surface of the wings, close to the trailing edge, and delayed static stall by suppressing back-flow velocities which amplify a beginning trailing edgeseparation. A dual-flap layout with a similar passive operation was reported by Bramesfeld et al. [14].

This study investigates the dynamic stall flow topology of an active spoilertype back-flow flap mounted on the suction side of a helicopter main rotorblade airfoil. The basic concept was patent-registered by Höfinger [15]. A numerical study was performed to estimate possible gains of the flap and to optimize its geometrical parameters, see Kaufmann et al. [16]. If the back-flow flap is attached to the suction side of the airfoil in a distance of 0.4 chord lengths to the leading edge, and deployed during dynamic stall, it will split up the large-scale stall vortex. A reduction of the corresponding $C_{m}$-peak of up to $34 \%$ was demonstrated. In a next step, wind tunnel experiments were conducted as a proof-of-concept. Therefore, the back-flow flap assembly and an active electromagnetic actuation system was built as a "retro-fit" kit for an existing wind tunnel model which uses the OA209 helicopter main rotorblade airfoil. The design of the flap setup is outlined by Opitz et al. [17,18], 
including a detailed description of the flap's kinematics and its flexure hinge. For dynamic stall conditions, the active back-flow flap showed very promising results as seen by surface-pressure measurements [19]. The deep-stall related pitching-moment peak was reduced by up to $25 \%$, whereas the maximum drag increased by only $2.5 \%$, and the lift characteristics remained approximately the same. It was also observed that in a self-actuated passive mode, in which the flap is only deployed through aerodynamic suction, light dynamic stall can be almost completely suppressed.

The focus of the current paper is a detailed evaluation of the deep-stall flow field over the suction side of the airfoil, which will be investigated using high-speed particle image velocimetry (PIV). The analysis allows a correlation between aerodynamic forces on the model and corresponding flow phenomena such as separation or dynamic stall development, illustrating the flap's method of operation.

\section{Experimental setup}

\subsection{Model geometry and test conditions}

An investigation of the pitch-oscillating helicopter main rotor-blade airfoil OA209 with a chord length $c=0.3675 \mathrm{~m}$ and a span of $1 \mathrm{~m}$ was conducted in the open test section of the closed-loop wind tunnel "1MG" at DLR Göttingen. The back-flow flap has a length of about $0.12 c$ and rotates around a flexure hinge located at $x / c=0.3$, see figures 1 and 2 . When fully withdrawn, the flap is placed in a recess of the airfoil's upper side providing a flush surface. The flap is operated either in active mode through an electromagnetic actuation system or in passive mode through aerodynamic suction. In both cases, a retention system limits the maximum opening angle to about $30^{\circ}$. A detailed description of the flap design and further details, for example the determination of the flap angle using Hall-effect sensors, is given by Opitz et al. [17, $18]$.

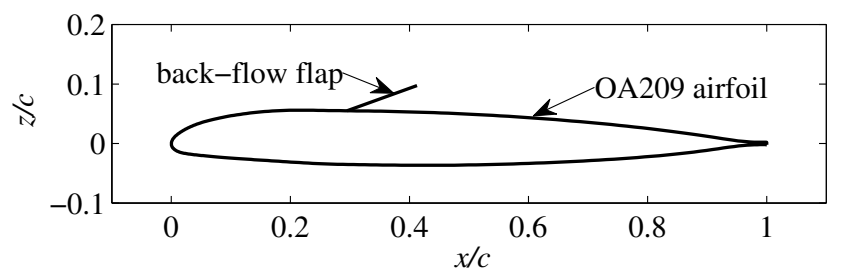

Fig. 1 Airfoil geometry

The model can be mounted into a test rig using a shaft protruding from both sides of the airfoil. The shaft is located at quarter chord position, and sinusoidal oscillations are applied by the rig through a controlled and geared 
electric motor. This hardware setup was successfully applied in preceding dynamic stall studies [20]. In the following, the notation " $\alpha=22^{\circ} \pm 8^{\circ}$ " refers to a sinusoidal oscillation of the angle of attack $\alpha$ with a mean value of $22^{\circ}$ and an amplitude of $8^{\circ}$. Per definition, the phase angle of the pitch oscillation is 0 at the minimum of $\alpha$ and $180^{\circ}$ at the maximum of $\alpha$.

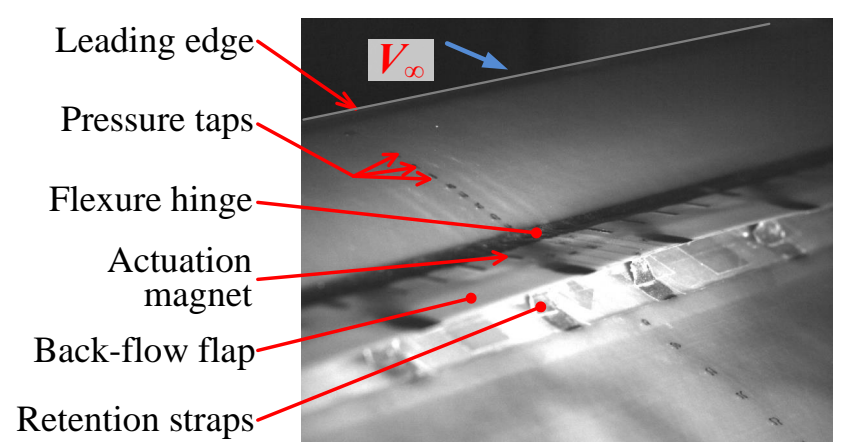

Fig. 2 High-speed camera image taken during the operation of the back-flow flap, adapted from ref. [19]

Table 1 summarizes the freestream and pitch motion parameters contemplated in this study. In particular, three different reduced pitch frequencies $k$ were considered, with a reference test case at $k=0.06(f=2.5 \mathrm{~Hz})$. The mean angle of attack and subsonic Mach number is chosen to be similar to the retreating side of a helicopter rotor in fast forward flight, and the results will be applicable to other subsonic Mach numbers. It is unclear whether the flow control using the back-flow flap will also be as effective for higher Mach numbers which have a shock-induced separation. The literature contains several examples of flow control which effectively makes this transition to the transonic flow regime $[21,22]$, as well as several which do not either from the formation of additional strong shocks [23] or too little power available leading to a lack of control authority [24], the latter of which will not be a problem for this kind of flap. It should be added that this flap can be held closed when the flow conditions for actuation are unfavorable [18].

Table 1 Freestream and pitch motion parameters

\begin{tabular}{lc}
\hline Freestream velocity, $V_{\infty}(\mathrm{m} / \mathrm{s})$ & 50 \\
Mach number, $M$ & 0.14 \\
Chord Reynolds number, Re & $1.1 \times 10^{6}$ \\
Pitch frequency, $f(\mathrm{~Hz})$ & $1.25, \mathbf{2 . 5 0}, 5.00$ \\
Reduced frequency, $k$ & $0.03, \mathbf{0 . 0 6}, 0.12$ \\
Pitch motion & $22^{\circ} \pm 8^{\circ}$ \\
Flap operation & active, taped (off) \\
\hline
\end{tabular}




\subsection{Particle image velocimetry}

A particle image velocimetry (PIV) setup was applied to evaluate the instantaneous velocity components $(u, w)$ in a measurement plane which is located in the midspan region of the model and perpendicular to the spanwise direction. The measurement plane was illuminated using a dual-cavity Quantronix Darwin Duo laser combined to a light sheet optics, see figure 3. The wave length of the laser light was $527 \mathrm{~nm}$, the repetition rate was set to $1 \mathrm{kHz}$, and the resulting pulse energy was around $30 \mathrm{~mJ}$. As tracer particles, aerosolized di-ethylhexyl-sebacate was used. The particle motion was captured using double-frame high-speed cameras of type pco.dimax, two cameras were installed to enhance the optical resolution. The cameras' fields of view focus on the forward and rearward half of the flow over the airfoil's suction side, with a small overlap in the mid-chord region. The particle motion during the separation time of both laser cavities, 14 ss, was evaluated using cross-correlation algorithms provided by the software "LaVision DaVis". An iterative multi-grid approach with circular final interrogation windows of $24 \mathrm{px}(=1.32 \% c)$ diameter was applied. Due to a window overlap of $75 \%$, the grid spacing of the resulting velocity fields is $0.33 \%$ c. Random cross-correlation errors during PIV evaluation can be estimated to about $0.1 \mathrm{px}$ [25]. With the given optical resolution of 4.95 $\mathrm{px} / \mathrm{mm}$ and the separation time of $14 \mu \mathrm{s}$, this results in a velocity error of $1.44 \mathrm{~m} / \mathrm{s}$ or $2.9 \% V_{\infty}$ affecting the instantaneous velocity distributions presented in this paper. Further additional error sources exist, such as calibration or timing errors. However for a well-prepared PIV setup, the correlation error will be the most significant error source, and is therefore a good indicator for the overall PIV uncertainty.

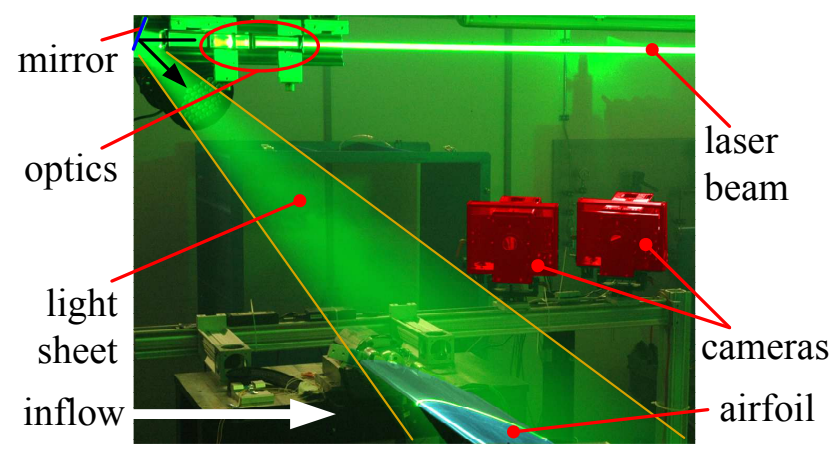

Fig. 3 PIV Setup 


\subsection{Data postprocessing}

A common reference target was used for the spatial calibration of both cameras, allowing for an automated stitching of the two measurement regions through DaVis. Additional postprocessing was accomplished using MATLAB. Since the calibration target was carefully positioned with respect to the airfoil, the center of the pitch oscillations is known in the coordinate system of the PIV records. For any pitch angle $\alpha$, the velocity fields can now be transformed into a model-fixed coordinate system $(x, z)$ with $x=0$ at the leading edge (also see figure 1). The airfoil contour is masked with an additional offset of $\Delta z=6.2 \mathrm{~mm}=1.7 \% c$ to account for strong laser reflections on the model's surface. For test cases with an actuated back-flow flap, also the flap surface and the corresponding shadow in the laser light sheet were removed from the results, creating a triangular masked-out region on top of the airfoil.

\subsection{Phase average and proper orthogonal decomposition}

A total of 5000 PIV double-images accounting for a time span of $5 \mathrm{~s}$ were taken for each test point, limited by the vast storage requirements of the image data. Since the image acquisition frequency of $1 \mathrm{kHz}$ is an integral multiple of the pitch frequencies $\{1.25 \mathrm{~Hz}, 2.50 \mathrm{~Hz}, 5.00 \mathrm{~Hz}\}$, the PIV measurements are inherently phase-locked with a resolution of $\{800,400,200\}$ images per cycle. An interpretation of instantaneous flow fields is difficult since large-scale structures are masked by small-scale turbulence or random events. Therefore, the phase-locked measurements enable a simple calculation of phase-averaged quantities. However, a total cycle count of $\{6.25,12.5,25\}$ per test point depending on the pitch frequency is too small to arrive at converged statistics. A possible solution is a sliding average filter over multiple phase angles. On the other hand, this procedure blurs or even eliminates short-term events, such as dynamic stall vortices.

The proper orthogonal decomposition (POD) provides a very powerful tool to identify spatio-temporal coherent patterns in a large dataset. In-depth discussions on the background of the POD approach are, for example, given in Refs. [26,27], here, only the basic principle is summarized. Any flow field $\boldsymbol{u}(\boldsymbol{x}, t)$ as part of a discrete-time series with $1 \ldots N$ samples (here: $N=5000$ ) can be expressed as:

$$
\boldsymbol{u}(\boldsymbol{x}, t)=\overline{\boldsymbol{u}}(\boldsymbol{x})+\sum_{m=1}^{N} a_{m}(t) \boldsymbol{\Phi}_{m}(\boldsymbol{x})
$$

Bold symbols represent non-scalar quantities, i.e., vectors or matrices. The flow field is decomposed into its time-averaged field $\overline{\boldsymbol{u}}$ plus a sum of $N$ terms. Each term consists of a time-varying scalar coefficient $a_{m}$ and an invariant eigenmode $\boldsymbol{\Phi}_{m}$, which can also be interpreted as a pseudo flow field. The contribution of the $m$ th eigenmode to the overall fluctuation energy is given 
by the corresponding eigenvalue $\lambda_{m}$ and its relative share $\lambda_{m} / \sum \lambda_{m}$. The POD has several appealing properties for flow analysis. For example, it can be used as a very efficient low-pass filter. Particularly, it can be shown that for a low-order reconstruction only using $N^{*}<N$ modes, there is no other decomposition of $N^{*}$ th order which approximates the original flow fields better than the POD.

By definition, the energy content of each mode (the mode's contribution to the total velocity fluctuations) decreases with increasing mode number. This can be seen from the fluctuation of the corresponding coefficient $a_{m}$, whereas the Frobenius norm of any eigenmode $\boldsymbol{\Phi}_{m}$ is equal to one. When analyzing time-resolved datasets, the temporal evolution of the low-order coefficients $a_{m}$ give information on the sequence of possible large-scale coherent flow structures as a function of time or phase.

Refs. [4,28] already demonstrated the application of the POD method in dynamic stall test cases. It was shown that the flow separation is responsible for a large part of the overall fluctuation level, and can therefore be tracked by means of the low-order eigenmodes of the POD analysis. It is argued that events characteristic for the initial phases of dynamic stall, for example the dynamic stall vortex, are also represented by corresponding eigenmodes. The current results will confirm these findings, but also reveal that an interpretation is less transparent for complex flow situations.

In the results section, the flow topology will be presented by means of a POD reconstruction of 15 th order using the phase-averaged coefficients $a_{1}$ to $a_{15}$. Since the choice of the reconstruction order is arbitrary, it will be shown that the resulting filtered flow fields capture the large-scale but shortlived dynamic stall events and filter out small-scale or aperiodic structures. A closer interpretation of the first modes and their relative energy will also be discussed. For the POD algorithm, any grid point taken into account must be valid throughout the entire pitch cycle. This means that for active flap cases, the masked-out region of the back-flow flap always corresponds to the maximum opening angle during the cycle, even if the flap is fully retracted at a given phase angle.

\subsection{Additional measurement techniques}

The wind tunnel model was equipped with a total of 40 fast-response pressure sensors of type Kulite XCQ-093, connected to pressure taps distributed in a chordwise section near the model's centerline. The corresponding distributions of the pressure coefficient $C_{p}$ can now be integrated, providing an estimate of the phase-averaged coefficients for lift $\left(C_{l}\right)$, pitching moment $\left(C_{m}\right)$, and pressure drag $\left(C_{d}\right)$. Also the surface area of the flap, its opening angle, and the static pressures below and above the flap were considered. A detailed description of the pressure measurements and conclusions regarding the dynamic stall behavior is given by Gardner et al. [19]. The pressure signals were sampled phase-locked at $20 \mathrm{kHz}$ over a timespan of $40 \mathrm{~s}$, providing a much better tem- 
poral resolution and a better statistical basis in comparison to the PIV results. The absolute errors of lift force and pitching moment measurements are estimated to be about $3 \%$ and $5 \%$ mainly due to discretization errors, but the relative errors between different test cases are lower than 1\% [19]. The data recorder of the pressure sensors was also used to acquire the signals of further equipment, providing a synchronization for the measurement of $\alpha$, the wind tunnel freestream parameters, PIV events like laser or camera trigger, the deflection angle of the back-flow flap, etc.

\section{Results}

\subsection{Reference test case with and without flow control}

In a first step, the effect of the back-flow flap is studied in detail for a reference test case with $V_{\infty}=50 \mathrm{~m} / \mathrm{s}$ and a pitching motion of $\alpha=22^{\circ} \pm 8^{\circ}$ at $2.5 \mathrm{~Hz}$. The large angles of attack were needed to compensate the wind tunnel effects of the open test section. The freestream parameters result in a Mach number of $M=0.14$ and a chord-based Reynolds number of $R e=1.1 \times 10^{6}$. A transition strip with a height of $152 \mu \mathrm{m}$ was applied to the suction side of the airfoil at $x / c=0.05$. It was shown by Gardner et al. [19] that the boundary layer tripping reduces the scatter of the results, but it does not affect the main conclusions with respect to the back-flow flap. Therefore, the current work focuses on a comparison of the flow structure between taped flap and actively deployed flap with tripped boundary layer.

The phase-averaged opening angle of the back-flow flap in the active test case is plotted as blue graph in figure 4 . The flap has a duty cycle of $25 \%$, and the begin of the opening was triggered to coincide with the pitching moment stall at a phase of $135^{\circ}$. There is a slight phase delay due to the actuation system and the flexure of the flap itself [19]. The blue error bars correspond to the cycle-to-cycle standard deviation. The flap's opening process takes place between phase angles of $145^{\circ}$ and $170^{\circ}$, and it will be shown later that this interval also covers entire dynamic stall vortex event. The corresponding standard deviation of the flap angle is below $1.7^{\circ}$ and small in comparison to the maximum opening angle of $25^{\circ}$. It is therefore assumed that the cycle-to-cycle differences of the flap opening have no significant influence on the presented results, even though no detailed study was conducted. During the subsequent fully separated flow at phase angles between about $170^{\circ}$ and $260^{\circ}$, the standard deviation increases up to $6^{\circ}$, and the phase-averaged opening angle shows larger fluctuations. No assessment of the flow field during this phase is presented in the current paper, since the effect of the back-flow flap is negligible.

The phase-averaged $C_{l}$ and $C_{m}$ distributions, see figures 5 and 6 for both taped and active flap, show clear indications of dynamic stall for phase angles between about $135^{\circ}$ and $315^{\circ}$ by means of reduced lift levels and a negative ("nose-down") pitching moment. The back-flow flap reduces the negative pitching moment peak at phase angles between $165^{\circ}$ and $170^{\circ}$ by about $21 \%$ 


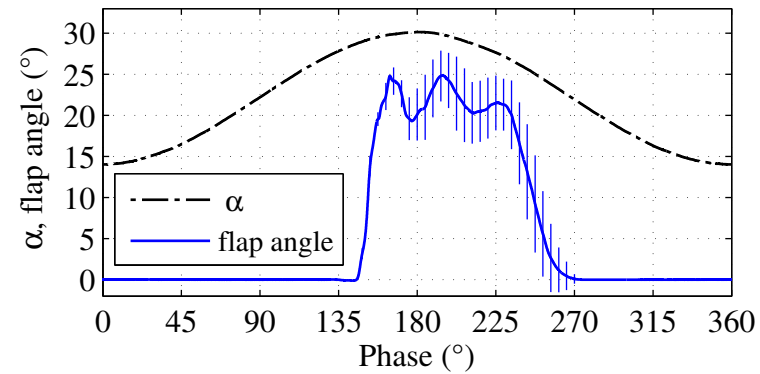

Fig. 4 Reference test case, $\alpha$ angle and flap angle with cycle-to-cycle standard deviation

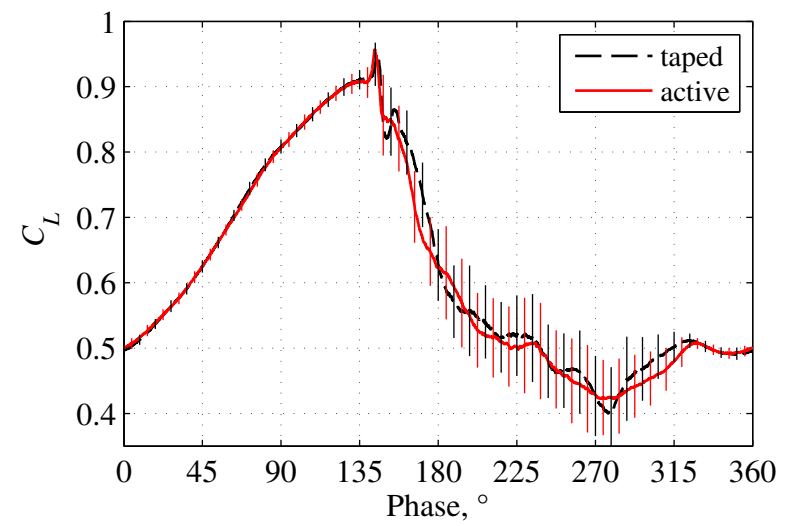

Fig. $5 C_{l}$ with cycle-to-cycle standard deviation for $\alpha=22^{\circ} \pm 8^{\circ}, k=0.06$

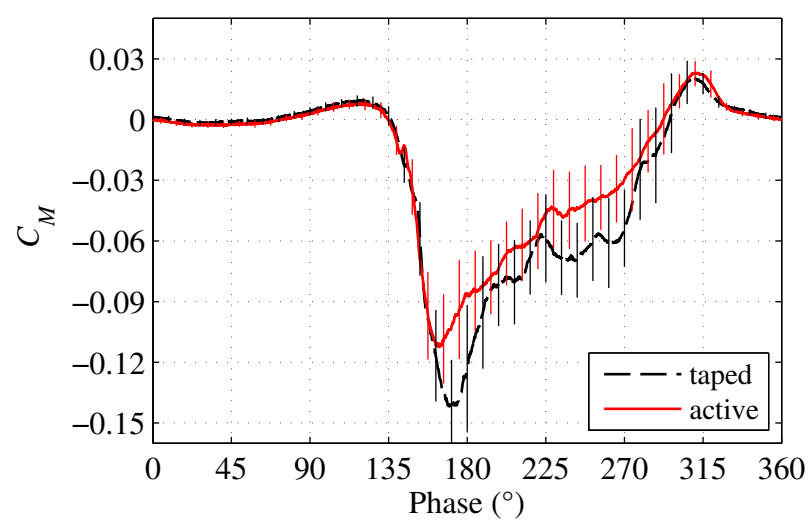

Fig. $6 C_{m}$ with cycle-to-cycle standard deviation for $\alpha=22^{\circ} \pm 8^{\circ}, k=0.06$

$\left(C_{m}=-0.113\right.$ versus $\left.C_{m}=-0.143\right)$, and retains this positive effect during the later phases of the dynamic stall process. In contrast to this, the flap's impact on the lift coefficient is almost negligible, and only a slight increase of about $1 \%$ regarding the maximum $C_{l}$-level is observed. 


\subsubsection{Pitching moment stall and initial lift stall}

In order to connect stall events of the flow to the corresponding forces and moments on the airfoil, the $C_{l^{-}}$and $C_{m}$-distributions are repeated for a smaller relevant phase range of $130^{\circ} \ldots 180^{\circ}$ in figure 7 .
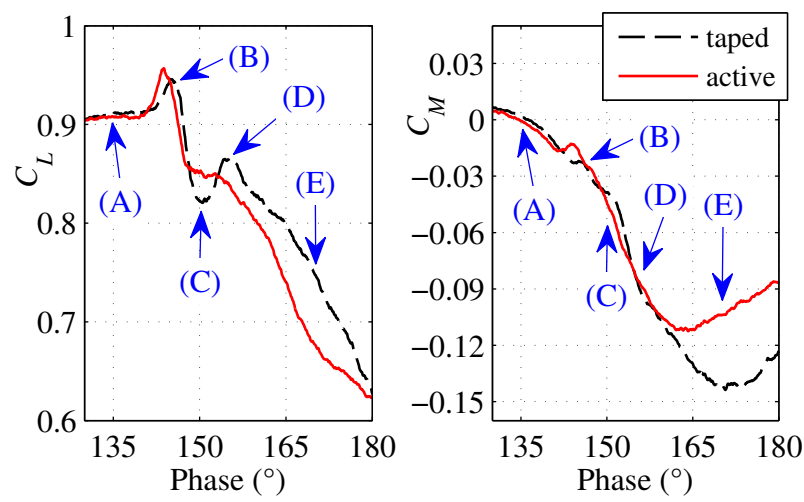

Fig. $7 C_{l}, C_{m}$ for $\alpha=22^{\circ} \pm 8^{\circ}, k=0.06$

During the largest part of the upstroke, the flow over the suction side of the airfoil is fully attached, and the back-flow flap of the "active flap"-configuration is retracted. The first indications of dynamic stall can be found by means of a beginning trailing edge separation, which takes place at a phase angle of $135^{\circ}$.

At this phase, the lift is still slightly increasing $\left(\partial C_{l} / \partial \alpha>0\right)$ but the pitching moment is rapidly dropping $\left(\partial C_{m} / \partial \alpha<0\right)$, see label $(\mathrm{A})$ in figure 7 . This indicates moment stall. The flow fields in figure 8 are colored by the magnitude of the in-plane velocity level $V_{p}$,

$$
V_{p}=\sqrt{u^{2}+w^{2}}
$$

and the corresponding flow direction is represented by streamlines. Instantaneous representations of the flow (figure 8, top) reveal turbulent flow structures developing in a thick boundary layer towards the trailing edge. These structures are filtered out in the phase-averaged POD of 15th order (figure 8, bottom) for two reasons: Small-scale structures are most probably aperiodic and therefore do not appear in a phase-averaged frame. Also, a reconstruction of higher order would be needed to accurately model these small (low-energy) patterns.

Following the argumentation of Mulleners and Raffel [28], the roll-up and merging of initial small-scale shear layer vortices will finally lead to the formation of a periodic large-scale event, the dynamic stall vortex. The initial stages of this process are hard to identify by means of instantaneous representations 


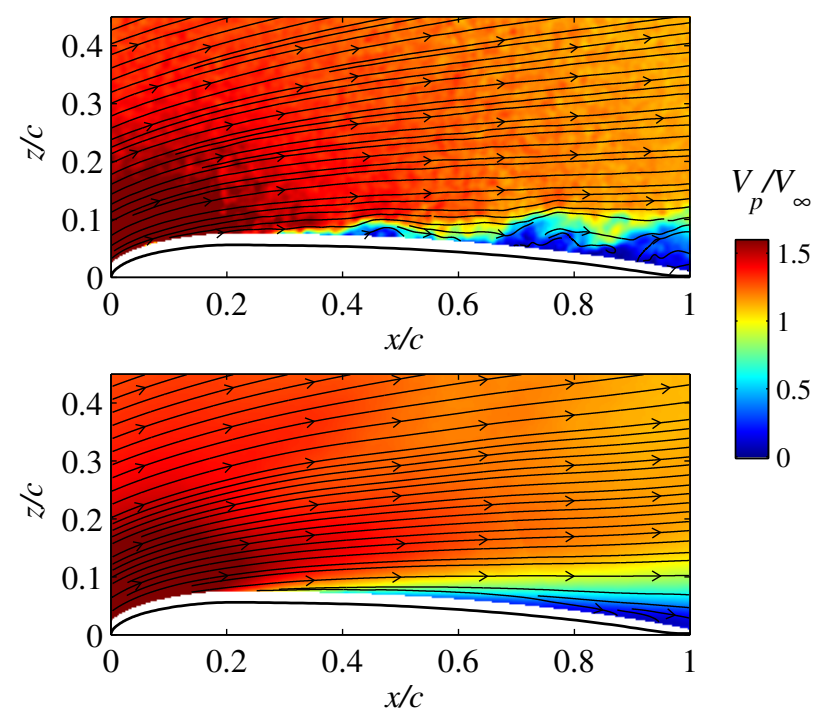

Fig. 8 Taped flap, phase $=135^{\circ}, C_{l}=0.91, C_{m}=0.0018$, instantaneous flow field (top) and POD reconstruction 15th order (bottom)

of the flow, for example see figure 9, top, for the taped flap case at a phase angle of $146^{\circ}$.

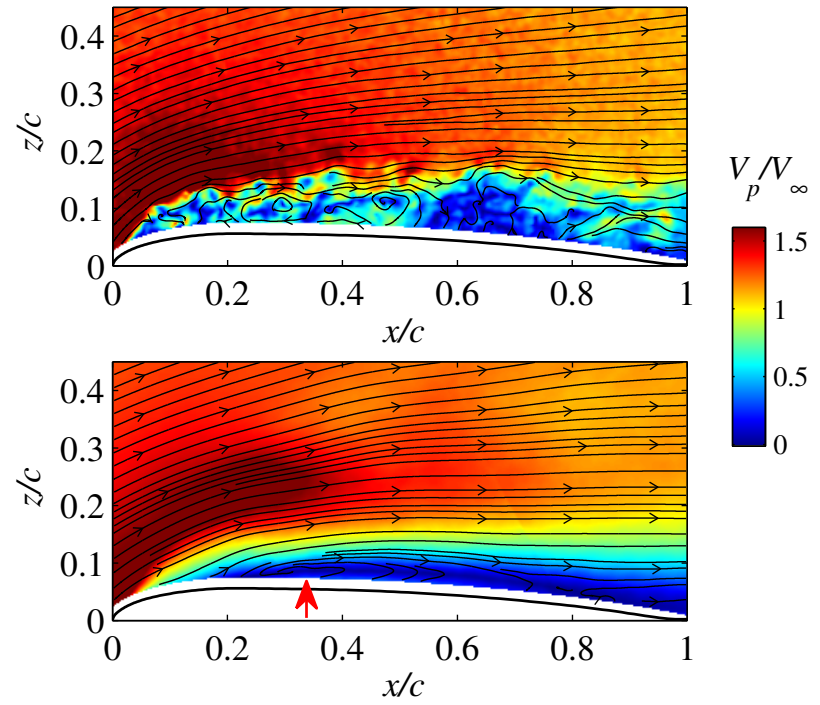

Fig. 9 Taped flap, phase $=146^{\circ}, C_{l}=0.93, C_{m}=-0.026$, instantaneous flow field (top) and POD reconstruction 15th order (bottom) 
The corresponding POD (figure 9, bottom) reveals a thin separation bubble stretching over a large central part of the airfoil's upper surface. The center of the circulating streamlines is at about $x / c=0.35$, see arrow marker, and this pattern will later evolve into the dynamic stall vortex. The external flow stays in close vicinity to the airfoil, it is further accelerated over the flat separation bubble. This also explains the sharp "spike" in the $C_{l}$ distribution, which reaches its global maximum (taped flap: $C_{l}=0.95$, active flap: $C_{l}=0.96$ ) at phase angles of about $144^{\circ}-146^{\circ}$, see label (B) in figure 7 . In this early stage of dynamic stall, it is hard to identify a possible influence of the back-flow flap in the PIV records. However, the very small opening angle of the flap and the similar $C_{l^{-}}$and $C_{d^{-}}$values for active and taped flap indicate that the differences between both test cases are small.

\subsubsection{Dynamic stall vortex}

For phase angles between about $150^{\circ}$ and $170^{\circ}$, the flow over the airfoil's suction side is governed by the dynamic stall vortex. The differences between the taped and the active flap are difficult to identify in instantaneous snapshots of the flow, but become apparent when analyzing the POD-filtered representations. For the taped flap at a phase of $150^{\circ}$, the vortex center is located at about $x / c=0.40$ and $z / c=0.13$, see figure 10 . The external flow successively detaches from the airfoil's surface, and the lift force assumes a local minimum of $C_{l}=0.82$, see label (C) in figure 7 . The vortex then moves downstream and away from the airfoil's surface. It is located at about $x / c=0.54$ and $z / c=0.23$ for a phase of $155^{\circ}$, see figure 11 . The POD-filtered flow shows a large backflow area stretching over almost the entire airfoil with peak values of up to $0.75 V_{\infty}$, the instantaneous velocities can be much higher. In this phase, the dynamic stall vortex evokes a second lift peak with a value of $C_{l}=0.86$, see label $(\mathrm{D})$ in figure 7 . The pitching moment is still rapidly dropping with increasing angle of attack.

At a phase angle of $149^{\circ}$, the opening angle of the active flap is still quite small, even though the flap's hall sensor $\left(\beta=3.7^{\circ}\right)$ probably underestimates the true value due to bending of the flap in the first stages of the opening process [19]. Nevertheless, its influence on the flow separation is notable, since the initial stall vortex forms further upstream in comparison to the taped flap case, see figure 12. According to the POD, the vortex center is located at about $x / c=0.29$ and $z / c=0.14$. The curvature of the streamlines in the vicinity of the flap indicates a second vortical structure downstream of the flap, inside of the masked-out region. This second vortex rapidly grows in size and is clearly visible at a phase angle of $154^{\circ}$ in both instantaneous and PODfiltered flow fields, see figure 13. The resulting separation bubble is of similar size in comparison to the taped flap case, but the back-flow velocity component over the airfoil is at least partly inhibited by the flap. Consequently, the $C_{l^{-}}$ distribution does not show a clear second lift peak as observed for the taped flap case, see figure 7 . 


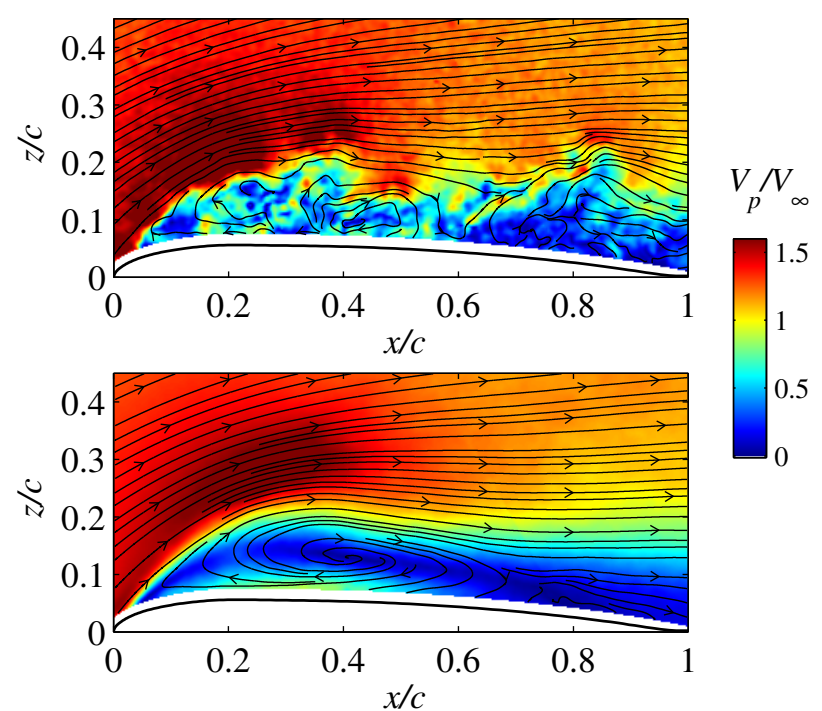

Fig. 10 Taped flap, phase $=150^{\circ}, C_{l}=0.82, C_{m}=-0.039$, instantaneous flow field (top) and POD reconstruction 15th order (bottom)

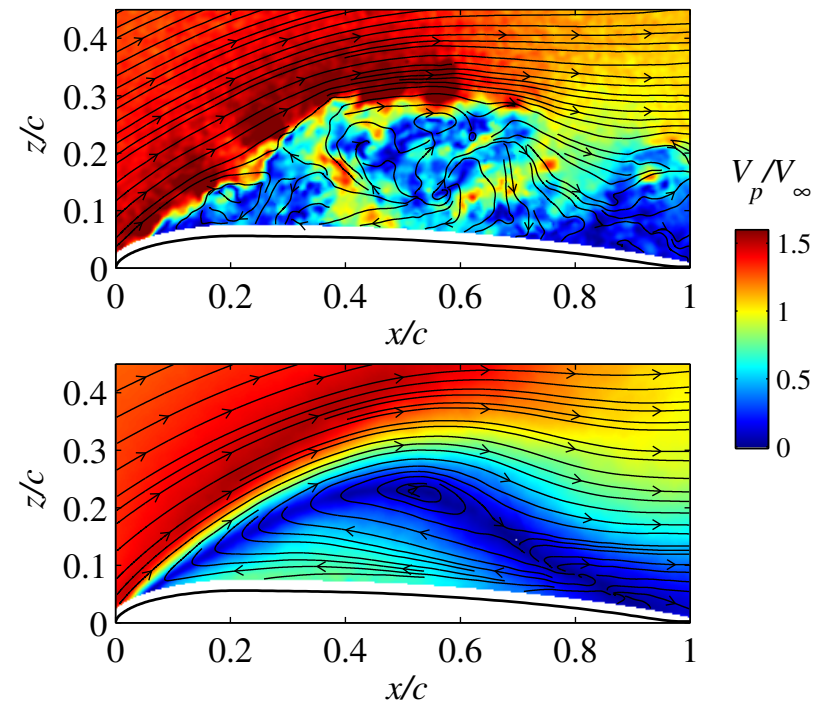

Fig. 11 Taped flap, phase $=155^{\circ}, C_{l}=0.86, C_{m}=-0.083$, instantaneous flow field (top) and POD reconstruction 15th order (bottom)

\subsubsection{Pitching moment peak, fully separated flow, reattachment}

The fully developed dynamic stall vortex, or the second vortex structure in case of the active flap, will convect downstream until reaching the area of the trailing edge. This induces a strong rear-loading and a negative pitching 


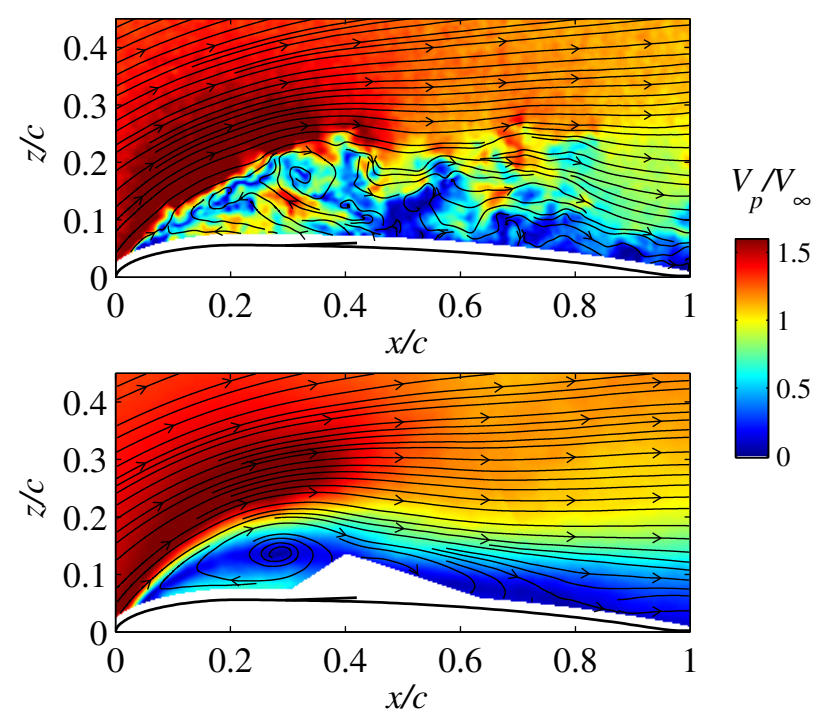

Fig. 12 Active flap $\left(\beta=3.7^{\circ}\right)$, phase $=149^{\circ}, C_{l}=0.85, C_{m}=-0.038$, instantaneous flow field (top) and POD 15th order (bottom)

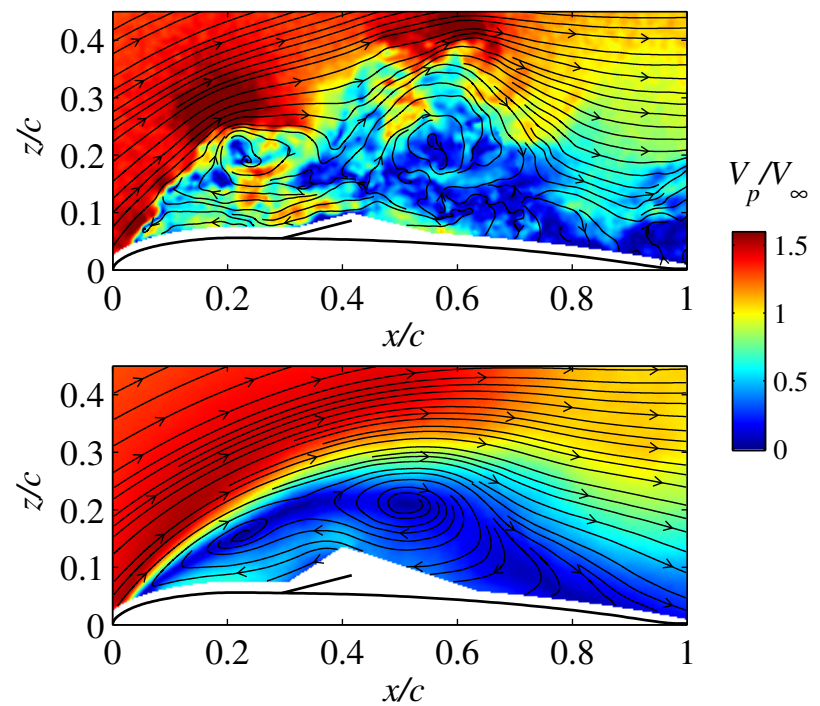

Fig. 13 Active flap $\left(\beta=15.5^{\circ}\right)$, phase $=154^{\circ}, C_{l}=0.84, C_{m}=-0.075$, instantaneous flow field (top) and POD reconstruction 15th order (bottom)

moment peak, which is observed at phases of $164^{\circ}$ for the active flap or $170^{\circ}$ for the taped flap. For the latter phase, the corresponding flow fields of both taped and active flap are shown in figures 14 and 15. Again, the active flap suppresses the back-flow along the airfoil's suction side, and the POD reveals that the stall vortex is still split up into two vortical structures located up- and downstream 

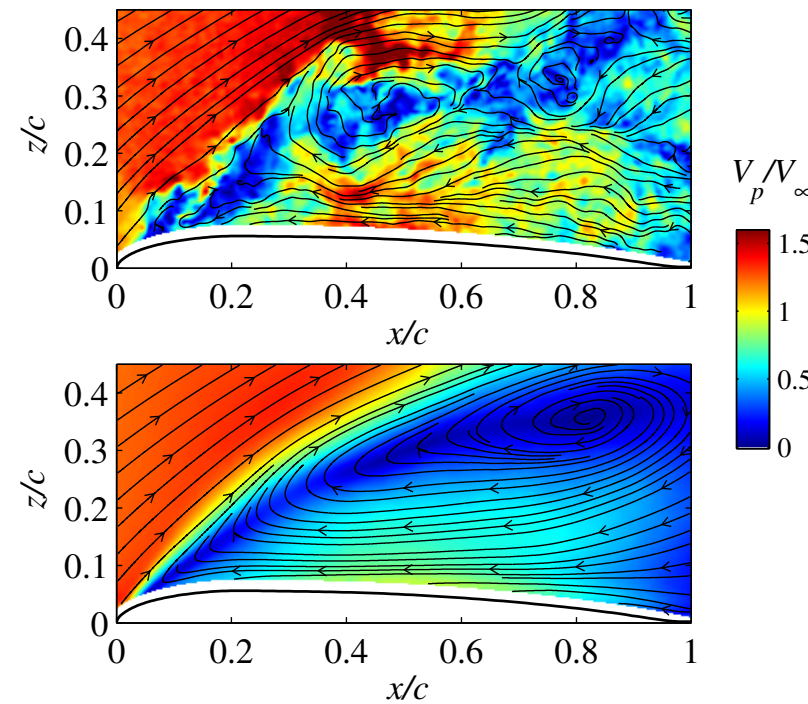

Fig. 14 Taped flap, phase $=170^{\circ}, C_{l}=0.75, C_{m}=-0.14$, instantaneous flow field (top) and POD reconstruction 15th order (bottom)

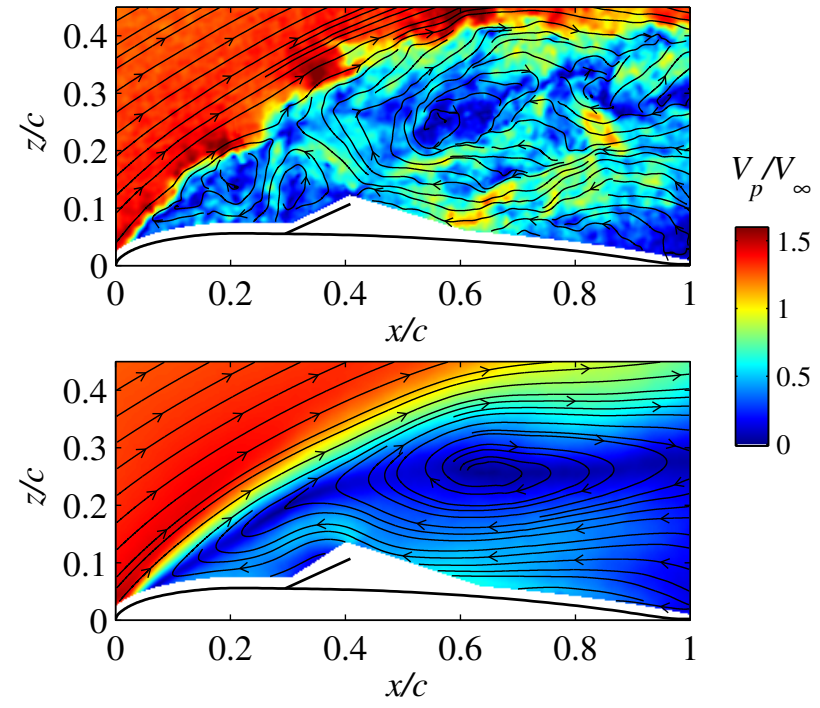

Fig. 15 Active flap $\left(\beta=23.4^{\circ}\right)$, phase $=170^{\circ}, C_{l}=0.68, C_{m}=-0.10$, instantaneous flow field (top) and POD reconstruction 15th order (bottom)

of the flap. Comparing the larger second structure of the active configuration to the dynamic stall vortex of the taped configuration, it is notably smaller, and located further upstream and closer to the airfoil. In summary, the flow 
fields illustrate the reduction of the phase-averaged pitching moment peak by about $21 \%$, see label (E) in figure 7, and also the flap's method of operation.

After the stall vortex has passed the trailing edge, the airfoil enters the fully separated state and the wake flow is much larger than the PIV's region of interest. The velocity distributions may still reveal large-scale vortices in the wake, but these structures are mainly aperiodic and lack large back-flow velocities. Towards the end of the cycle, the flow successively reattaches to the suction side of the airfoil beginning from the leading edge. No systematic differences between the active and taped flap are identified for the discussed reference test conditions.

\subsubsection{POD modes and coefficients}

The POD eigenvalues $\lambda_{m}$ show that the first 15 POD modes used for the low-pass filter in the preceding sections account for $91 \%$ (taped flap) and $89 \%$ (active flap) of the overall velocity fluctuation energy during the pitch cycles. The distributions of the relative energies $\lambda_{m} / \sum \lambda_{m}$ are given in figure 16 . The

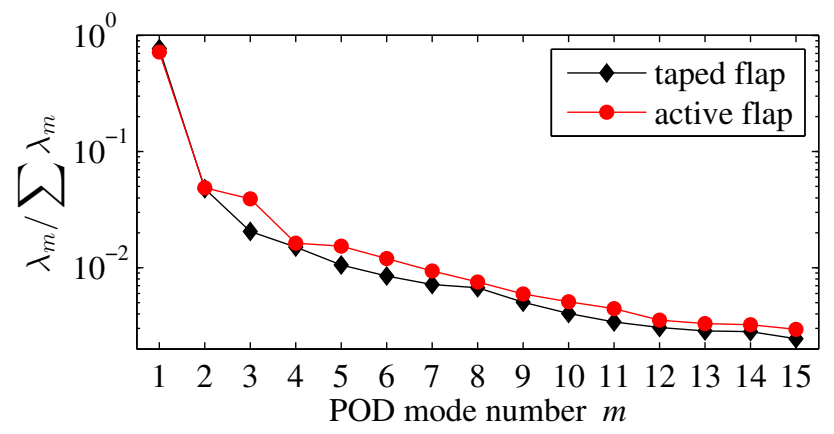

Fig. 16 Relative energy of POD modes $1 \ldots 15$, reference case

first eigenmode has a dominant influence on the flow, with relative energies of $77 \%$ (taped flap) and $71 \%$ (active flap). This mode represents the alternation between separated and attached flow. The corresponding pseudo flow field $\boldsymbol{\Phi}_{\mathbf{1}}$ for the active flap is depicted in figure 17 as $(u, w)$-vectors, with only every 20 th vector in both coordinate directions shown. Due to the normalization of $\boldsymbol{\Phi}$, the absolute magnitude of the vectors has no significance. The spatial layout of the vectors shows the border between external flow and separated flow starting in the lower left corner, sketched as a red dash-dotted line in figure 17. The taped flap eigenmode 1 (not shown) is qualitatively similar. Mulleners and Raffel [28] arrive at the same conclusion regarding the POD representation of separated flow. It is noted that in ref. [28], the average flow field is not subtracted before POD and therefore appears as mode 1, shifting the subsequent modes by one with respect to the numbering in this paper. 


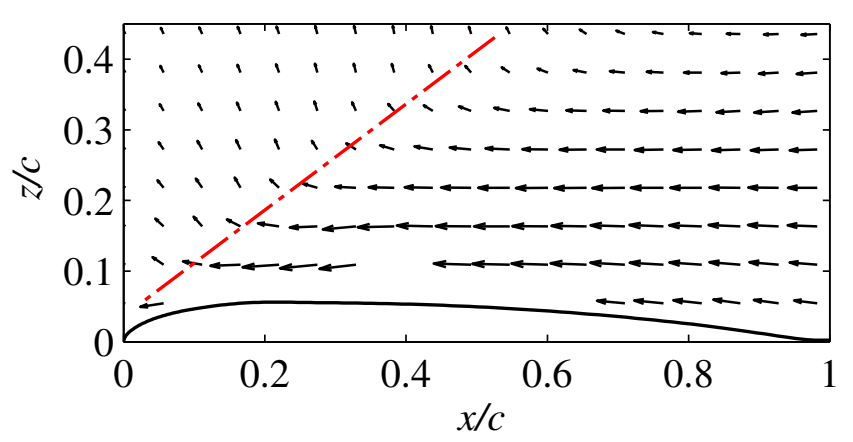

Fig. 17 POD Mode 1, reference case with active flap, the dash-dotted line illustrates the border of the wake for stalled conditions

The temporal coefficient of mode $1, a_{1}$, switches between a low level representing attached flow and a high level representing separated flow, see figure 18. The transition from an attached flow state to a separated flow state occurs between about $135^{\circ}$ and $175^{\circ}$, which coincides with the dynamic stall processes. There are no relevant differences between the taped and active flap configurations, meaning that the flap does not alleviate or even prevent mode 1 from forming.

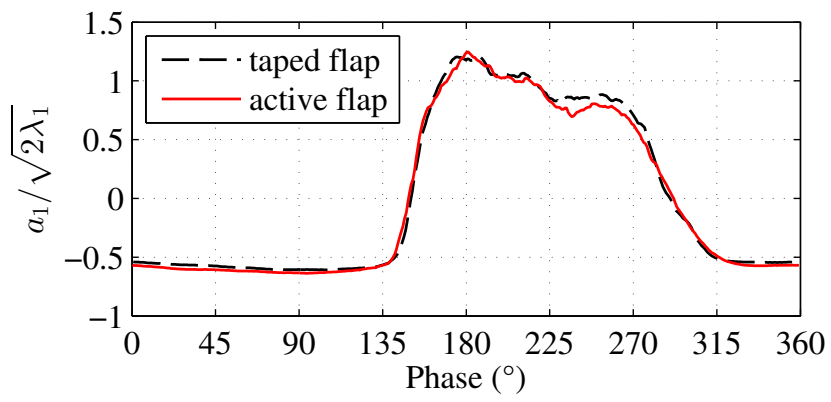

Fig. 18 Phase-averaged normalized coefficients of mode 1, reference case

Regarding the subsequent modes, the flap evokes a change in the modal structure of the flow. Mode 2 has a contribution of about $5 \%$ for both the taped and active flaps. The layout of the mode can be described as a vortical structure above the airfoil, located at about $x / c=0.55$ (taped flap, figure 19) or $x / c=$ 0.75 (active flap, figure 20). This eigenmode can be related to the dynamic stall vortex [4,28], since a superposition with the average flow field and eigenmode 1 results in a large-scale vortical structure and a bubble-like separated area above the airfoil. It must be noted that a reconstruction of 2 nd order cannot reproduce complex aspects of dynamic stall, such as the convection of the stall vortex towards the trailing edge. The inclusion of additional modes is then required. Nevertheless, the phase-averaged coefficient $a_{2}$ for the taped 


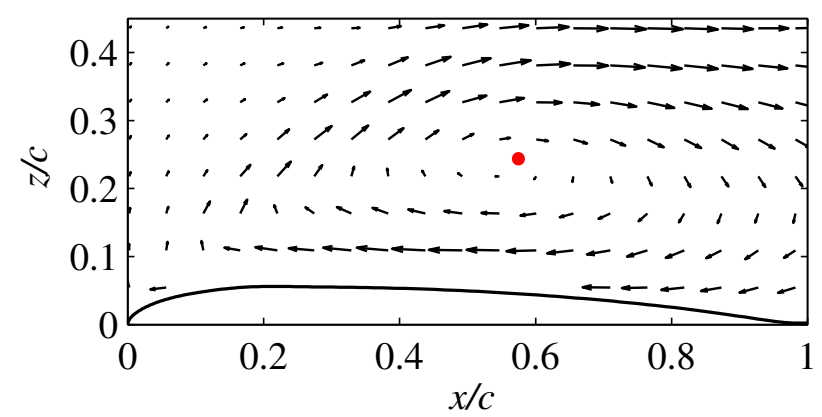

Fig. 19 POD mode 2, reference case, taped flap, the dot marks the center of rotation

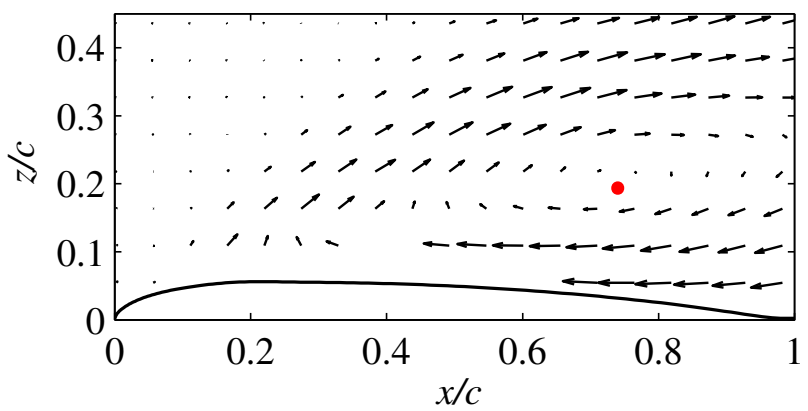

Fig. 20 POD mode 2, reference case, active flap, the dot marks the center of rotation

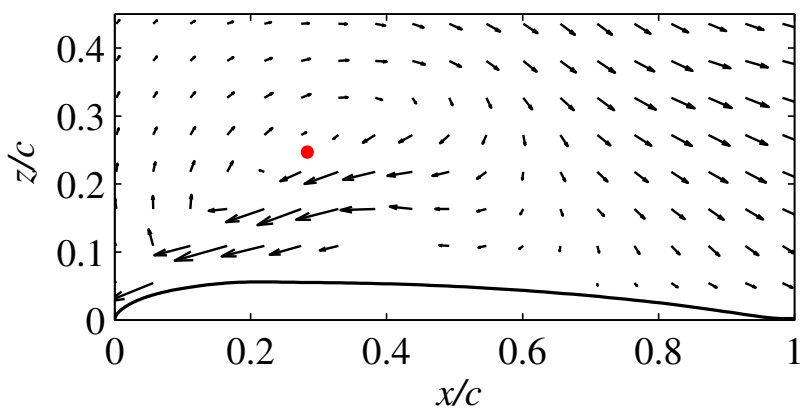

Fig. 21 POD mode 3, reference case, active flap, the dot marks the center of rotation

flap shows a distinct peak at a phase of $154^{\circ}-155^{\circ}$, see label (D) in figure 22 . This event coincides with the second lift peak of the $C_{l}$-distribution, label (D) in figure 7 , and the flow topology shown in figure 11. In contrast, the coefficient $a_{2}$ of the active flap-configuration has much lower values and no distinct peak at this phase, underlying the suppression of the stall vortex.

The relative contribution of mode 3 to the active flap case (about $4 \%$, see figure 16) is almost twice as large as the contribution to the clean airfoil case (about 2\%). The evolution of the corresponding coefficients $a_{3}$ reveal a distinct dynamic stall-related maximum for the active flap-case, see label (D) in figure 23. In contrast, the taped flap-coefficient assumes several smaller 


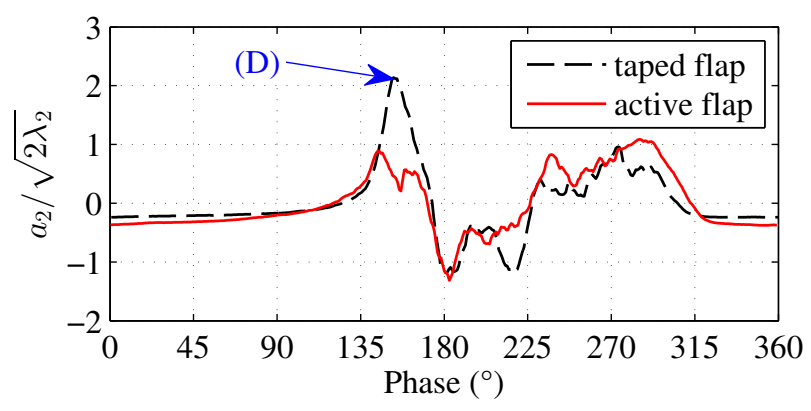

Fig. 22 Phase-averaged normalized coefficients of mode 2, reference case

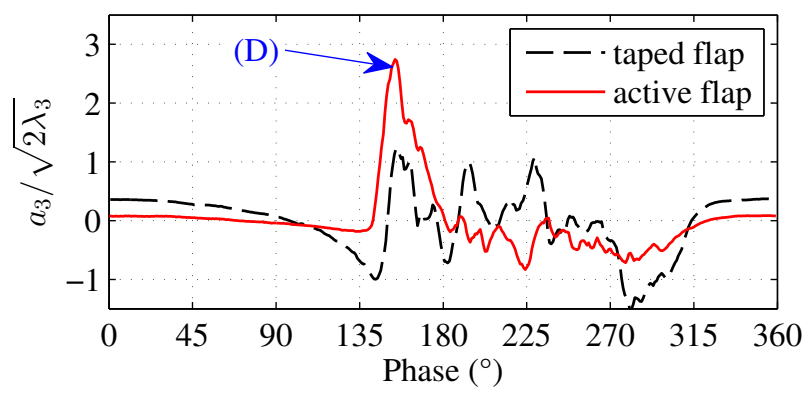

Fig. 23 Phase-averaged normalized coefficients of mode 3, reference case

maxima throughout the entire separated flow phase, indicating that this mode and its spatial structure are of minor relevance to the dynamic stall process. The structure of the active flap's eigenmode 3 , see figure 21 , consists of a second vortical structure upstream of the vortex of eigenmode 2 , located at about $x / c=0.3$. A POD reconstruction of 3rd order at a phase of $154^{\circ}$ ("mean flow plus separation plus two vortices") is shown in figure 24. This low-order reconstruction is very similar to the higher-order reconstruction in figure 13 due to the strong influence of the coefficients $a_{2}$ and $a_{3}$, which in combination represent the split-up dynamic stall vortex due to the flap deployment. In many other situations, for example in the early stage of stall vortex development at a phase of $150^{\circ}$, a higher-order representation is mandatory for an appropriate description of the flow.

In summary, the structure of the flow topology can be attributed to the POD coefficients $a_{m}$, the eigenvalues $\lambda_{m}$, and the eigenmodes $\boldsymbol{\Phi}_{\boldsymbol{m}}$. The interpretation of individual modes is difficult for complex flow fields due to the possible superposition of two or more modes. For the reference case in active flap mode, the flow separation and the split-up of the stall vortex by means of eigenmodes 2 and 3 was demonstrated. 
3.2 Variation of the pitching frequency

As part of a parameter study, three different reduced pitching frequencies $k=\{0.03,0.06,0.12\}$ were investigated. Figures 25 and 26 show the phase-

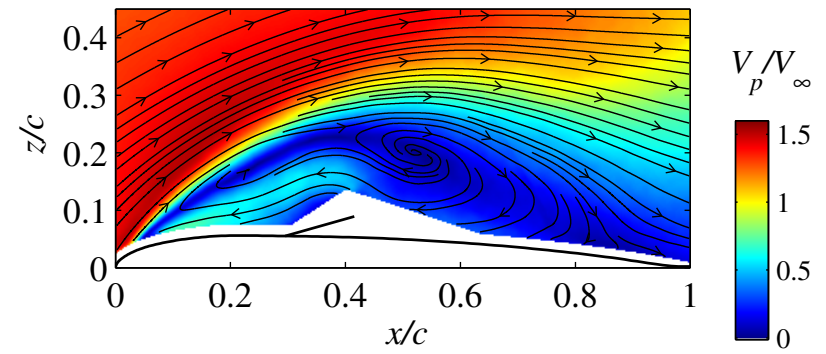

Fig. 24 Active flap $\left(\beta=15.5^{\circ}\right)$, phase $=154^{\circ}, C_{l}=0.84, C_{m}=-0.075$, POD reconstruction 3rd order

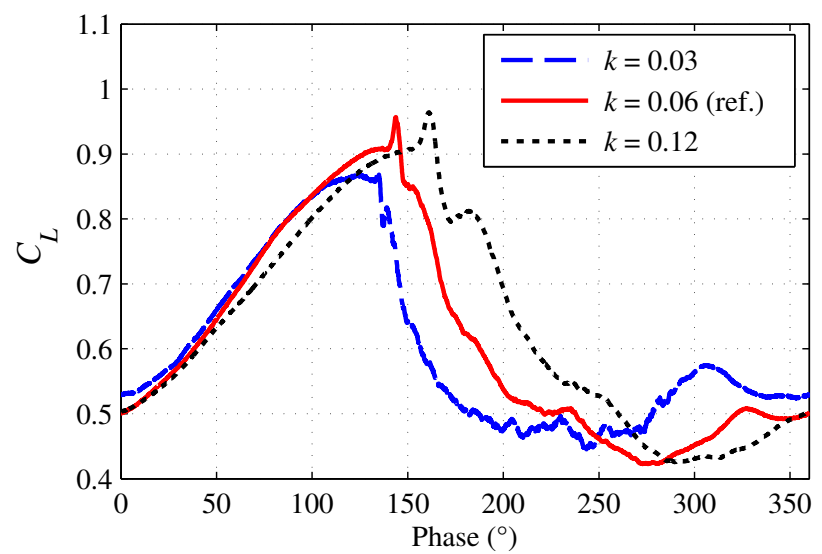

Fig. 25 Phase-averaged $C_{l}$ for $\alpha=22^{\circ} \pm 8^{\circ}$ and different pitching frequencies, active flap

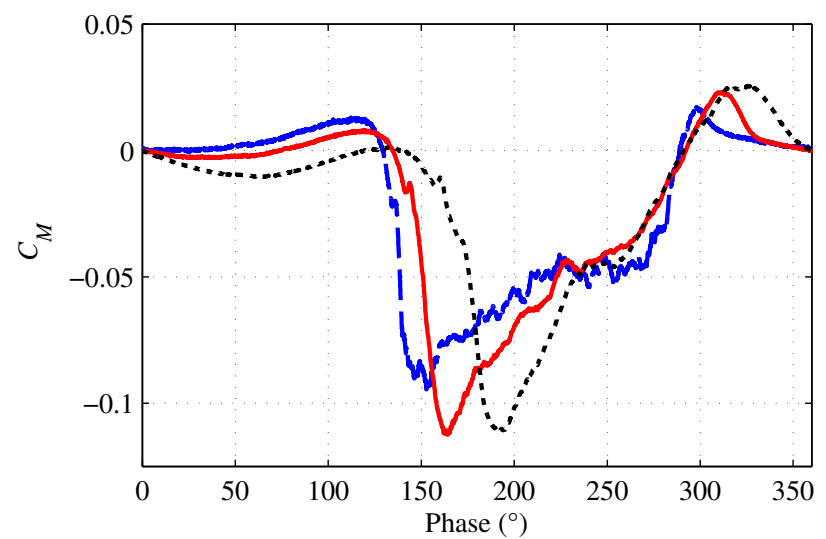

Fig. 26 Phase-averaged $C_{m}$ for $\alpha=22^{\circ} \pm 8^{\circ}$ and different pitching frequencies, active flap 
averaged lift and pitching moment coefficients for the corresponding active flap cases. With increasing frequency, the stall events are delayed towards later phase angles as a consequence of an increasing hysteresis. For the lowest frequency, both the maximum $C_{l}$-value and the negative $C_{m}$-peak are reduced in comparison to the higher frequencies. Comparing the active flap configuration to the corresponding taped flap cases (not shown), a reduction of the negative $C_{m}$-peak by $\{25 \%, 21 \%, 12 \%\}$ is observed. At least under these conditions, it seems that the effectiveness of the back-flow flap deteriorates with higher pitch frequencies. A possible explanation is given by the opening behavior of the flap. The opening process takes place in a constant time window, which relates to an increasing phase window when reducing the pitching period [19]. It is also possible that higher pitching frequencies require different flap positions.

Regarding the POD results it must be noted that due to the fixed PIV acquisition rate, a higher pitching frequency $k$ results in a lower phase resolution but a larger number of samples at a given phase angle. The dynamic stall takes place during about one tenth of the entire cycle (e.g. $135^{\circ}$ to $170^{\circ}$ for $k=0.06)$. At the highest frequency of $k=0.12$, this interval is still covered by 20 data points, which is sufficient to capture the large temporal gradients. The low cycle count for the lowest frequency of $k=0.03$ mainly affects the fully separated flow state, where large-scale aperiodic events result in a stronger fluctuation of the POD coefficients.

The phase-averaged POD coefficients of the first mode, $a_{1}$, are given in figure 27. For all frequencies, the first mode corresponds to the flow separation similar to figure 17, and the relative contribution $\lambda_{1} / \sum \lambda_{m}$ of this mode is $\{75 \%, 72 \%, 68 \%\}$. The evolution of the coefficient $a_{1}$ confirms the increasing hysteresis with increasing frequency, since the transition from attached flow to separated flow (and vice versa) is shifted towards later phase angles. Also, the steepness of the rise in the first mode decreases, indicating that the flow separation takes longer with respect to the phase angles. When plotted against time instead of phase (not shown), the steepness of the rise in mode 1 is similar for all frequencies.

An unexpected behavior was found for the subsequent modes of the highest pitch frequency of $k=0.12$. The eigenmode with a vortical structure upstream of the flap, similar to figure 21, has a larger relative energy $(5.3 \%)$ than the eigenmode with the vortical structure downstream of the flap, similar to figure $20(4.7 \%)$. As a result, the mode numbers are switched in comparison to the cases with $k=0.03$ and $k=0.06$. Even though this implies that the pitching frequency has an effect on the modal structure the POD, a conclusive explanation for this behavior could not be determined. Figure 28 shows the third-order coefficients $a_{3}$ for $k=0.03$ and $k=0.06$ together with the secondorder coefficient $a_{2}$ for $k=0.12$. For all cases, a clear peak can be identified, representing the split-up of the dynamic stall vortex as discussed in the preceding section. Again, the steepness of the peak and its position is altered by the frequency variation. The phase angles of the peaks in figure 28 are $\left\{139^{\circ}\right.$, $\left.154^{\circ}, 176^{\circ}\right\}$. Apart from the hysteresis and differences in the relative modal 


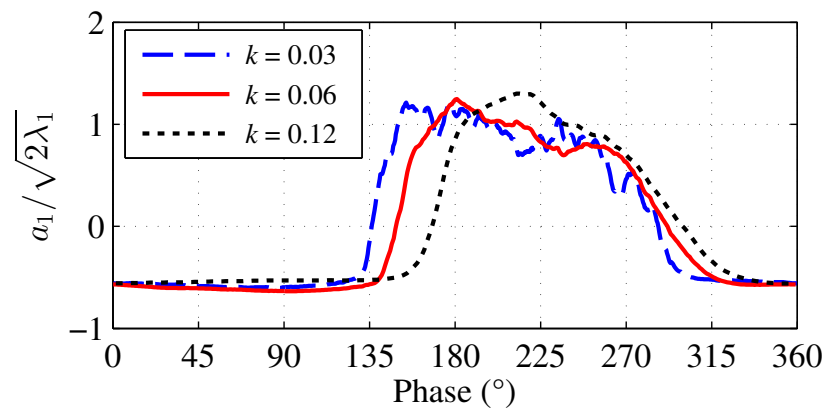

Fig. 27 Phase-averaged normalized coefficients of mode 1, different pitching frequencies, active flap

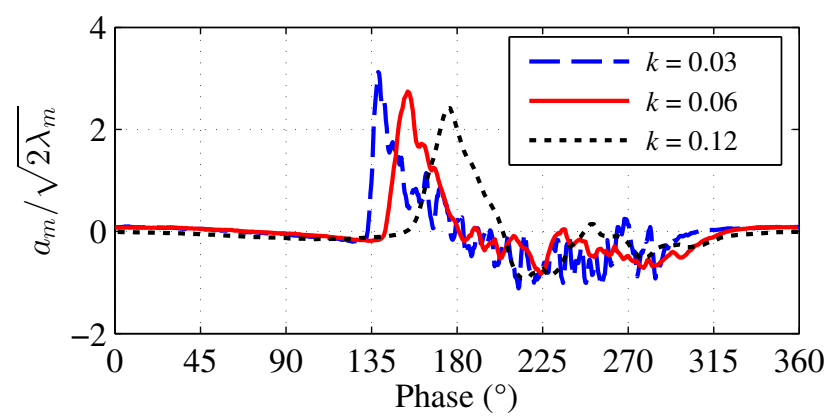

Fig. 28 Phase-averaged normalized coefficient $a_{3}$ for $k=0.03$ and $k=0.06, a_{2}$ for $k=0.12$, active flap

energy, the back-flow flap's general method of operation is unchanged by the different pitching frequencies.

\section{Conclusions}

An experimental study of a pitching helicopter main rotor-blade airfoil was conducted, investigating a back-flow flap intended for dynamic stall alleviation. The flow topology was characterized using high-speed particle image velocimetry. For a deep-stall reference test case, the evolution of the dynamic stall vortex was studied in detail for both active and taped flap configurations. Large-scale flow events were identified through a phase-averaged proper orthogonal decomposition (POD) and related to the lift and moment coefficients calculated from the pressure distribution. The flap suppresses back-flow velocity components in the vicinity of the airfoil's suction side and splits the dynamic stall vortex into two separate vortical structures, which are located up- and downstream of the flap. As a consequence, the pitching moment peak is reduced by $21 \%$. No significant influence on the lift force or the flow separation was observed for the discussed deep-stall test case. The effect of the 
back-flow flap can be described by means of the low-order eigenmodes and the temporal coefficients of the POD. A study of different pitching frequencies revealed the frequency-dependent hysteresis effects. The effectiveness of the back-flow flap is reduced at higher pitching frequencies, and the modal structure of the POD slightly changes. Future studies should concentrate on the light-stall behavior, for which a passively actuated flap showed promising results regarding stall suppression and lift characteristics as seen by pressure measurements [19].

\section{References}

1. McCroskey, W. J., Carr, L. W., McAlister, K. W.: Dynamic stall experiments on oscillating airfoils. AIAA J 14(1), 57-63 (1976). doi: 10.2514/3.61332

2. McCroskey, W. J.: The phenomenon of dynamic stall, NASA TM-81264 (1981).

3. Carr, L. W., McAlister, K. W., McCroskey, W. J.: Analysis of the development of dynamic stall based on oscillating airfoil experiments. NASA TN-D-8382 (1977).

4. Joubert, G., Le Pape, A., Heine, B., Huberson, S.: Deployable vortex generator dynamic stall alleviation through experimental and numerical investigations. J Am Helicopter Soc 58(3), 1-13 (2013). doi: 10.4050/JAHS.58.032005

5. Le Pape, A., Costes, M., Richez, F., Joubert, G., David, F., Deluc, J.-M.: Dynamic stall control using deployable leading-edge vortex generators. AIAA J 50(10), 2135-2145 (2012). doi: $10.2514 / 1 . J 051452$

6. Heine, B., Mulleners, K., Joubert, G., Raffel, M.: Dynamic stall control by passive disturbance generators. AIAA J 51(9), 2086-2097 (2013). doi: 10.2514/1.J051525

7. Greenblatt, D., Wygnanski, I.: Dynamic stall by periodic excitation, Part: NACA 0015 parametric study. J Aircraft 38(3), 430-438 (2001). doi: 10.2514/2.2810

8. Greenblatt, D., Nishri, B., Darabi, A., Wygnanski, I.: Dynamic stall control by periodic excitation, Part 2: Mechanisms. J Aircraft 38(3), 439-447 (2001). doi: 10.2514/2.2811

9. Post, M. L., Corke, T. C.: Separation control using plasma actuators: Dynamic stall vortex control on oscillating airfoil. AIAA J 44(12), 3125-3135 (2006). doi: 10.2514/1.22716

10. Chandrasekhara, M. S., Wilder, M. C., Carr, L. W.: Unsteady stall control using dynamically deforming airfoils. AIAA J 36(10), 1792-1800 (1998). doi: 10.2514/2.294

11. Gerontakos, P., Lee, T.: Dynamic stall flow control via a trailing-edge flap. AIAA J 44(3), 469-480 (2006). doi: 10.2514/1.17263

12. Meyer, R. K. J.: Experimentelle Untersuchungen von Rückströmklappen auf Tragflügeln zur Beeinflussung von Strömungsablösung. PhD Thesis, Technical University of Berlin (2000). ISBN: 3898202054

13. Meyer, R., Hage, W., Bechert, D. W., Schatz, M., Knacke, T., Thiele, F.: Separation sontrol by self-activated movable flaps. AIAA J 45(1), 191-199 (2007). doi: 10.2514/1.23507.

14. Bramesfeld, G., Maughmer, M. D.: Experimental investigation of self-actuating, uppersurface, high-lift-enhancing effectors. J Aircraft 39(1), 120-124 (2002). doi: 10.2514/2.2905.

15. Höfinger, M.: Rotor blade with integrated passive surface flap. Patent US9193456 B2 (2015).

16. Kaufmann, K., Gardner, A. D., Richter, K.: Numerical investigations of a back-flow flap for dynamic stall control. In: Dillmann, A., Heller, G., Krämer, E., Kreplin, H.-P., Nitsche, W., Rist, U. (Eds.) New Results in Numerical and Experimental Fluid Mechanics IX, pp. 255-262. Springer, Heidelberg (2014). doi: 10.1007/978-3-319-03158-3_26

17. Opitz, S., Gardner, A. D., Kaufmann, K.: Aerodynamic and structural investigation of an active back-flow flap for synamic stall control. CEAS Aeronautical J 5(3), 279-291 (2014). doi: 10.1007/s13272-014-0106-3

18. Opitz, S., Kaufmann, K., Gardner, A.: An active back-flow flap of a helicopter rotor blade. Advances in Aircraft and Spacecraft Science 1(1), 69-91 (2014). doi: 10.12989/aas.2014.1.1.069

19. Gardner, A. D., Opitz, S., Wolf, C. C., Merz, C. B.: Reduction of dynamic stall using a back-flow flap. CEAS Aeronautical J 8(2), 271-286 (2017). doi: 10.1007/s13272-017-0237-4 
20. Merz, C. B., Wolf, C. C., Richter, K., Kaufmann, K., Raffel, M.: Experimental investigation of dynamic stall on a pitching rotor blade tip. In: Dillmann, A., Heller, G., Krämer, E., Wagner, C., Breitsamter, C. (Eds.) New Results in Numerical and Experimental Fluid Mechanics X, pp. 339-348. Springer, Heidelberg (2016). doi: 10.1007/978-3-319-27279-5_30

21. Gardner, A. D., Richter, K., Mai, H., Neuhaus, D.: Experimental Investigation of Air Jets to Control Shock-Induced Dynamic Stall. J Am Helicopter Soc 59(2), 1-11 (2014). doi: 10.4050/JAHS.59.022003

22. Mai, H., Dietz, G., Geissler, W., Richter, K., Bosbach, J., Richard, H., de Groot, K.: Dynamic Stall Control by Leading Edge Vortex Generators. J Am Helicopter Soc 53(1), 26-36 (2008). doi: 10.4050/JAHS.53.26

23. Martin, P., Wilson, J., Berry, J., Wong, T., Moulton, M., McVeigh, M.: Passive Control of Compressible Dynamic Stall. 26th AIAA Applied Aerodynamics Conference, Honolulu, HI, USA, AIAA 2008-7506 (2008). doi: 10.2514/6.2008-7506

24. Min, B.-Y., Lorber, P. F., Berezin, C. R., Wake, B. E., Scott, M. W.: Numerical Study of Retreating Side Blowing Concept for a Rotor in High Speed Flight. AHS 73rd Annual Forum, Fort Worth, TX, USA (2017).

25. Raffel, M., Willert, C. E., Wereley, S. T., Kompenhans, J.: Particle Image Velocimetry: A Practical Guide, 2nd ed., pp. 131-176, Springer-Verlag Berlin (2007). doi:10.1007/9783-540-72308-0

26. Sirovich, L.: Turbulence and the dynamics of coherent structures pt I-III, Q Appl Math 45(3), 561-590 (1987). doi: 10.1090/qam/910462

27. Berkooz, G., Holmes, P., Lumley, J. L.: The proper orthogonal decomposition in the analysis of turbulent flows, Annu Rev Fluid Mech 25, 539-575 (1993). doi: 10.1146/annurev.fl.25.010193.002543

28. Mulleners, K., Raffel, M.: The onset of dynamic stall revisited, Exp Fluids 52(3), 779793 (2012). doi: 10.1007/s00348-011-1118-y 